\title{
Understanding Non-Invasive Glucose Level Sensing Using Raman Scattering to Propose Novel Schemes
}

\author{
Aldo Di Costanzo Mata, Luis Cortez González, Daniel Ceballos Herrera, \\ Arturo Castillo Guzmán, Romeo Selvas Aguilar \\ Universidad Autónoma de Nuevo León (UANL), Facultad de Ciencias Físico Matemáticas, \\ Nuevo León, Mexico
}

Aldo.DiCostanzo@gmail.com

\begin{abstract}
For Healthcare professionals is important to measure the glucose level in diabetes patients in order to prevent any systematical failure. For this manner it is important to understand the social and economic advantages of developing non-invasive sensing methods. The main objective is to use the Raman Scattering to develop schemes that predicts the value of glucose level on patients using the Source and Stokes wavelengths energies signals.
\end{abstract}

Keywords: non-invasive glucose level sensing, Raman scattering.

\section{Introduction}

Public healthcare is a medullar objective in every nation and society. An impact on public healthcare generates low statistics on Labor Force and in consequence it generates low economic rates. Increasing constantly, diabetes has become a very common condition in world's population, over 347 million people globally present diabetes [9]. $80 \%$ of diabetes deaths occur on the low and middle income countries [10].

In 2010, global healthcare expense for prevention and treatment of diabetes was valued around \$376 Billion US Dollars. By 2030 the expected expense would be $\$ 490$ Billion US Dollars [4]. In regards of the Biosensors market, by 2004 the glucose biosensors represented the $85 \%$ of the world's market valued in \$5 Billion US Dollars [6]. This factors open opportunities to develop devices that decreases the diabetes healthcare cost increasing biosensors market for good.

Non-invasive diagnostic methods provide advantages that improve the healthcare procedures and minimize the need of interventions. In the imaging area it's possible to explore patient internal structures to develop prosthesis that perfect fit, plan surgeries or detect the exact position of a tumor. In our case, sensing blood glucose level with optical devices opens the possibility of constant sensing, maintains biological system intact and prevents tissue exposure, frequent nerve damage, pain and recurrent purchase of disposables [2]. 


\section{$2 \quad$ Fundamentals}

\subsection{Diabetes}

Diabetes mellitus is defined as the group of metabolic diseases that present high levels of glucose in the blood. It is caused by a lack of production or high resistance from the tissues to insulin. Glucose levels are managed by the insulin-glucose feedback system via pancreatic hormones insulin and glucagon. During the interprandial period (time between meals) almost all glucose is used by the brain and the insulin-glucose feedback system prevents the Pancreas to produce insulin [3].

Glucose is mainly presented on its $\alpha$-D-glucose form and doesn't require any protein or other component to travel trough the bloodstream [8]. Regular glucose levels oscillates around 75-95 mg/dl during a normal day. A high glucose level causes cell dehydration. A prolonged exposure to high glucose concentrations causes tissue damage in peripheral nerves and vascular cells which promotes heart attacks, stroke, renal failure, blindness, etc [3].

\subsection{Raman Scattering}

Used to study and identify a sample (like glucose) in the electromagnetic spectrum, illustrated on figure 1, the Raman Scattering is a non-linear inelastic process where a molecular medium is pumped with a wpump frequency and then another lower frequency emerges; this new frequency is known as Stokes or $\omega$ Stokes. The Stokes frequency is presented when the molecule absorbs the wpump, that increases the energy level of a particle in ground state to a virtual state, then the particle decreases to a vibrational state emitting the $\omega$ Stokes, the rest of the energy is dissipated by the molecule vibration [1]. If the particle decreases to the ground state, the emitted frequency is equal to the wpump, this elastic effect it's known as Rayleigh scattering. If a particle is in vibrational state, then increases to a virtual state to decrease to a ground state, the new scattered frequency is known as Anti-Stokes or8 $\omega$ Anti-Stokes [5].

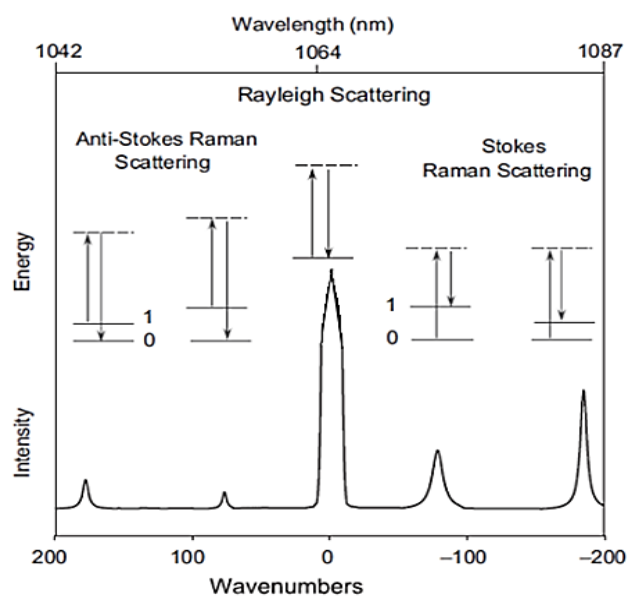

Fig. 1. Raman Scattering and Spectrum example. 


\section{Experimental Principles \& Proposal}

Our proposal is based on pulsioximeter principle, observed on Figure 2, as a preliminary model to obtain the desired interface. Finger vascular bed is pumped with two wavelengths $(660 \mathrm{~nm}$ and $940 \mathrm{~nm})$ and the logarithmic relationship between them delivers measurements that predict the oxygen saturation level on blood [2]. Our approach lies on the capability to implement photodetectors for the Rayleigh and Stokes wavelengths for the $\alpha$-D-glucose and to use the proper laser wavelength and intensity to provoke desired stokes emission. Collected and filtered data will be processed to implement a statistical method to predict the Blood Glucose Level.

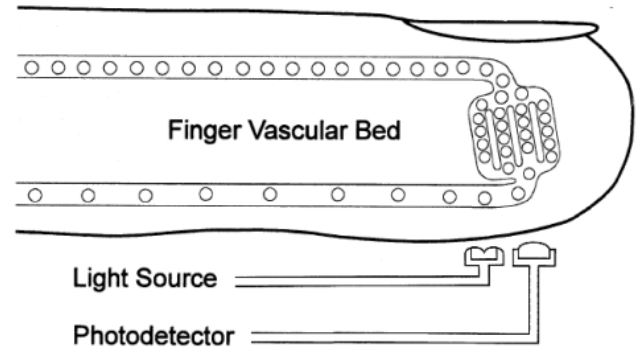

Fig. 2. Pulsioximeter Scheme.

A promising recent Raman spectroscopy applied on skin, spectrum shown on Figure 3 , used a $785 \mathrm{~nm}$ continuous laser radiation at $400 \mathrm{~mW}$ and a Shamrock SR-163 spectrograph coupled to a $-90^{\circ} \mathrm{C}$ cooled (for a better curve response) iDus DU-401 Detector. The results presented a 0.83 accuracy using a R2 coefficient of determination (Raman vs. Capillary Glucose), showing a mean relative deviation of $13.9 \pm 11.4 \%$ [7].

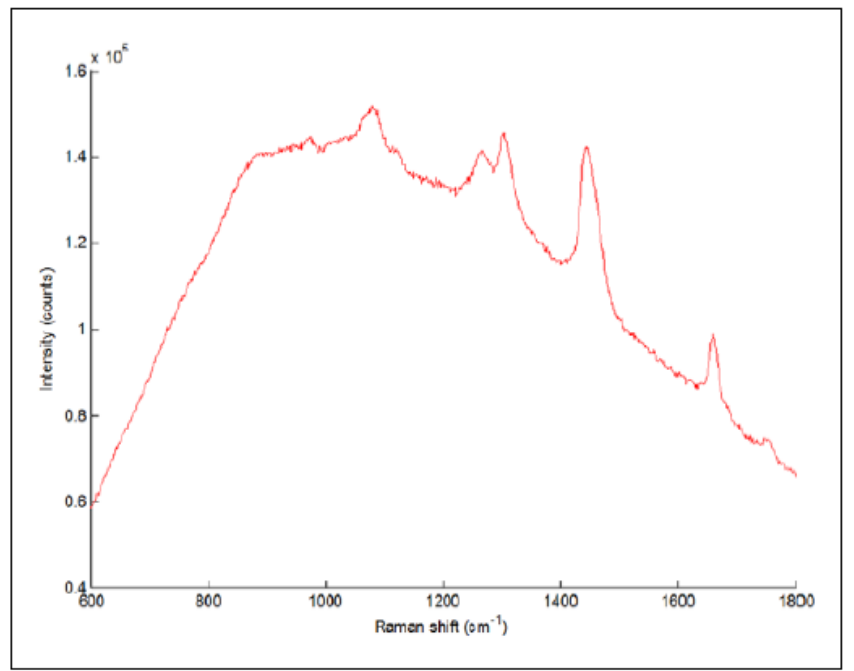

Fig. 3. Raman Spectrum of Human Skin Tissue. 
The main proposal, on Figure 4, is a circuit that consists on a Pump Laser and two detectors, one to detect the Pump wavelength or Rayleigh scattered light and the other to detect the Stoke's wavelength.

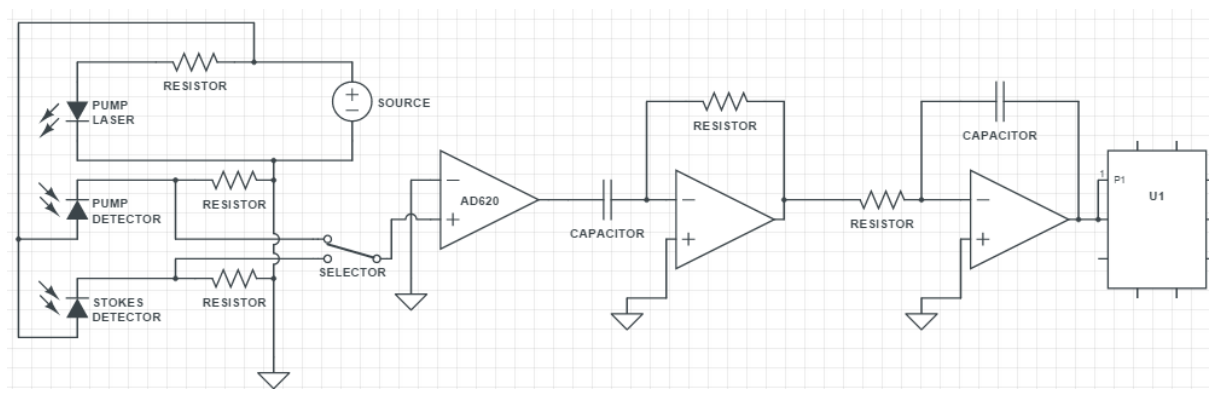

Fig. 4. Proposed circuit model.

An expected weak signal from each detector will be amplified by an instrumentation amplifier (Op. Amp. AD620). Then a Band-pass filter will be needed to remove the DC components and the non-desired electrical noise $(50 / 60 \mathrm{~Hz})$. After this step our signals can be converted and analyzed by a computer or microcontroller.

\section{Conclusions}

The proposal is based on the ideal scenario that we know the specific wavelengths (Pump, Rayleigh \& Stokes) energies (For $\alpha$-D-glucose) needed to detect, in order to apply the specific optical and electronical instrumentation to measure the signals without analyzing a considerable portion of the spectrum. Obvious investigation and experimentation processes are pending to develop. A device with these characteristics will occupy little space so it could be portable, cheaper if compared with a spectrum analyzer and easy to repair because the components can be replaced if the physical design allows it.

On regards Diabetes, a device with these characteristics would provide a diverse number of applications for healthcare treatment for example: Distance continuous monitoring, an alert system for patients and healthcare professionals, Smartphone applications for a better response in case of glucemic incident (GPS position + alert system), etc. This may lead to a lower diabetes healthcare cost and an increase on the economic activities related to biosensors, development, network applications, software, etc.

Acknowledgments. Authors want to thank to all collaborators and persons who supported this project with their knowledge and time. Special thanks to CONACYT via scholarship CVU/Becario: 627802/332756.

\section{References}

1. Agrawal, G. P.: Nonlinear fiber optics. Academic press (2007) 
Understanding Non-Invasive Glucose Level Sensing Using Raman Scattering to Propose Novel ...

2. Carvajal, R. A. E.: Propuesta de Tesis para la Maestría en Ciencias de la Ingeniería Electrónica con Especialidad en Electrónica. Biosensores, Monterrey Institute of Technology and Higher Education (2008)

3. Hall, J. E., Guyton, A. C.: Textbook of medical physiology. Elsevier Spain (2011)

4. International Diabetes Federation.: The Economic Impacts of Diabetes. Available at: http://www.idf.org/diabetesatlas/economic-impacts-diabetes (2009)

5. Larkin, P.: Infrared and Raman spectroscopy; principles and spectral interpretation. Elsevier (2011)

6. Newman, J. D., Turner, A. P.: Home blood glucose biosensors: a commercial perspective. Biosensors and Bioelectronics, DOI: 10.1016/j.bios.2004.11.012, 20(12), pp. 2435-2453 (2005)

7. Scholtes-Timmerman, M. J., Bijlsma, S., Fokkert, M. J., Slingerland, R., van Veen, S. J.: Raman Spectroscopy as a Promising Tool for Noninvasive Point-of-Care Glucose Monitoring. Journal of diabetes science and technology, DOI: 10.1177/1932296814543104 (2014)

8. Voet, D., Voet, J. G., Pratt, C. W.: Fundamentals of biochemistry: life at the molecular level. Editorial Médica Panamericana (2006)

9. World Health Organization.: Diabetes. Media Centre. Fact sheet $\mathrm{N}^{\circ} 312$. Available at: http://www.who.int/mediacentre/factsheets/fs312/en/ (2010)

10. World Health Organization.: 10 Facts about Diabetes. Diabetes. Available at: http://www.who.int/features/factfiles/diabetes/facts/en/index7.html 